\title{
Distinguishing Meteoritic Nanodiamonds from Disordered Carbon Using Atom-Probe Tomography
}

\author{
J. B. Lewis, ${ }^{1 *}$ D. Isheim ${ }^{2}$ C. Floss, ${ }^{1}$ and D. N. Seidman ${ }^{2}$
}

${ }^{1}$ Laboratory for Space Sciences, Physics Department, Campus Box 1105, One Brookings Drive, Washington University, St. Louis, MO 63130

${ }^{2}$ Center for Atom-Probe Tomography, and Dept. of Materials Science and Engineering, 2200 North Campus Drive, Northwestern University, Evanston, IL 60208

*jlewis@physics.wustl.edu

Abstract: We have analyzed atom probe tomography reconstructions of disaggregated meteoritic material containing nanodiamonds and disordered carbon to determine whether these phases formed in the solar system or whether they predate the solar system and were formed in supernovae or the interstellar medium. We developed a method to distinguish between these two carbonaceous phases in $<100 \mathrm{~nm}$ diameter aggregates using the ratios of various native and contaminant molecular species. We find variations in measured ${ }^{12} \mathrm{C} /{ }^{13} \mathrm{C}$ ratios between the two phases that suggest hydrides form more readily during field evaporation of the disordered $\mathrm{C}$ than the nanodiamonds.

Keywords: meteoritic nanodiamonds, atom probe tomography (APT), element ratios, isotope ratios, presolar grains

\section{Introduction}

Nanodiamonds are found in primitive meteorites by dissolving the host material in acid and conducting size separations on the residue [1]. The nanodiamonds account for only about half of the carbon in the residue [2], and the remainder is a disordered carbon phase with primarily $\mathrm{sp} 2$ bonding, probably glassy carbon [3]. These nanodiamond acid residues also contain an anomalous mixture of xenon isotopes, Xe-HL, enriched in heavy and light isotopes, believed to be produced only in Type II (core collapse) supernova explosions [1]. These data suggest that at least some of the acid residue material is composed of presolar grains-particles that condensed around supernovae and late-type stars and survived the formation of the sun and solar system to be incorporated into primitive solar system material such as chondrites, which are stony meteorites that have not experienced significant alteration by melting or icing and contain chondrules, that is, round grains of different minerals [4]. However, the ratios of stable carbon and nitrogen isotopes in bulk nanodiamond acid residues are consistent with terrestrial values [5], a puzzling observation since the Xe appears to be of supernova origin. The ratio of stable isotopes is the best indicator of whether material is presolar, as any material formed from solar gas will have a ${ }^{12} \mathrm{C} /{ }^{13} \mathrm{C}$ ratio within a small range of values near $90 / 1$, whereas presolar materials, including material from different supernovae, have ${ }^{12} \mathrm{C} /{ }^{13} \mathrm{C}$ ratios with ranging orders of magnitude from $1 / 1$ to $1000 / 1$, depending on the source. Transmission electron microscopy studies of the nanodiamonds show that they are on average less than $3 \mathrm{~nm}$ across, each containing around 2000 atoms [6]. Detailed studies of various types of presolar grains, which correlate with the observation and modeling of late-type stars, supernovae, and the interstellar medium, have yielded new information about these environments. However, meteoritic nanodiamonds and fragments of disordered carbon are so small that understanding their formation and alteration histories is limited by technical capabilities. Prior studies of the nanodiamond acid residues have measured millions or billions of nanodiamonds at a time.

It is possible that a subset of the acid residue is presolar, and the rest formed in the solar system with the terrestrial carbon and nitrogen from the solar material diluting anomalous signals from the presolar fraction to such a degree that it goes undetected in bulk measurements of millions of nanodiamonds. In this article we use atom probe tomography (APT) to measure the ${ }^{12} \mathrm{C} /{ }^{13} \mathrm{C}$ ratio of smaller fractions of acid residue than previously characterized [7-9]. We cannot study Xe with this approach because its concentration is too low for us to expect detection of even a single meteoritic Xe atom in samples of our size. An important advance
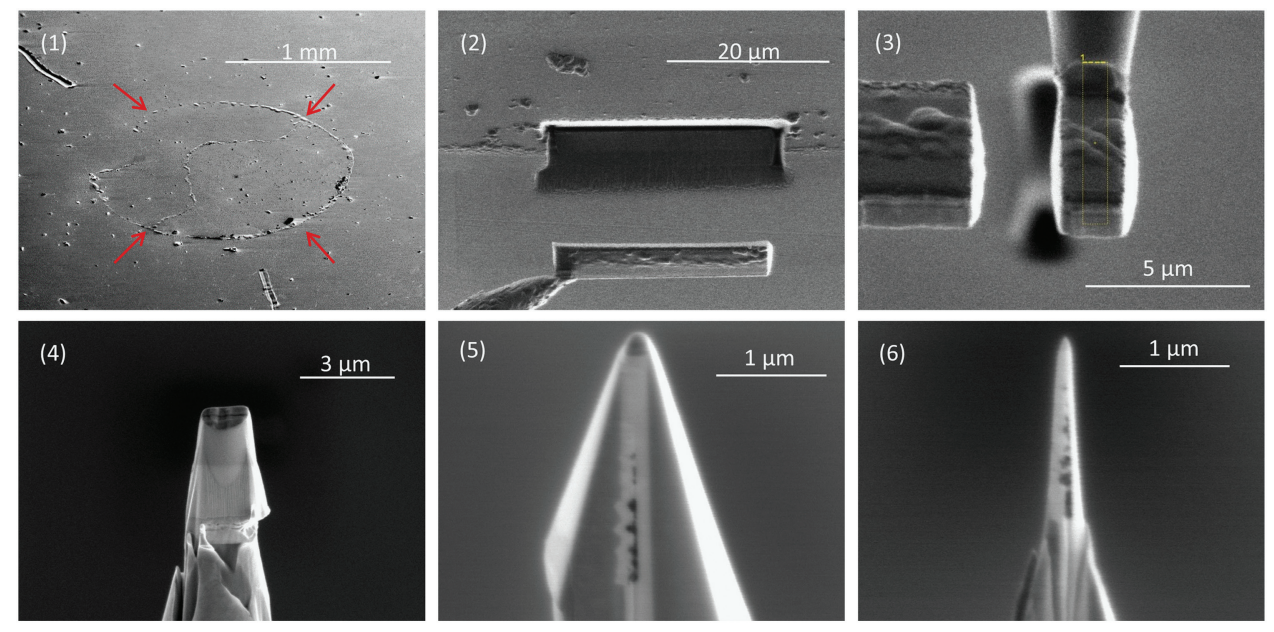

Figure 1: Sample preparation for APT. (1) A multilayer containing a circular deposit of acid residue (arrows), which presses up the covering Pt layer. The residue contains nanodiamonds and disordered carbon separated from the carbonaceous chondrite Allende by acid dissolution and size and density separations. A small droplet of water with suspended acid residue is placed on a Pt-coated substrate to make the deposit, which is then covered in a second layer of sputter-deposited Pt. (2) A FIB liftout of a $25 \mu \mathrm{m}$ long, $5 \mu \mathrm{m}$ wide undercut region of the multilayer. (3) A slice of the rotated multilayer attached to a $2 \mu \mathrm{m}$ diameter micropost using Pt deposition. (4) Annular milling by FIB produces a conical shape. (5) Additional FIB milling from four different angles produces a pyramid shape while preserving the Pt patch between the slice of multilayer and the micropost. (6) Final annular milling produces a nanotip with an apex of well under $100 \mathrm{~nm}$ in radius. 
in this research would be the discovery of a method to distinguish carbon isotopes of diamond from those of amorphous carbon in order to determine if diamonds, disordered carbon, or both contain a presolar fraction. The development of such a method is the topic of this article.

\section{Materials and Methods}

To prepare nanotips for APT we embedded a layer of acid residue between two layers of ion-beam sputter-deposited platinum and used focused ion beam (FIB) milling to create small radius $(<100 \mathrm{~nm})$ nanotips of the multilayer, with the plane of the acid residue running along the long axis through the nanotip (Figure 1). The nanotip shape is required so that high voltage in the atom probe will generate a high enough electric field to evaporate ions from the apex of the nanotip.

We used a LEAP 4000x straight-flight-path atom probe to collect time-of-flight spectra from the samples. Including data sets reported earlier $[7,9]$, we analyzed a total of 36 data sets from samples of acid residue from the Allende chondrite meteorite and 26 standard data sets from detonation nanodiamonds [10]. We detected in the acid residue clusters high in carbon-namely $\mathrm{C}_{1}, \mathrm{C}_{2}, \mathrm{C}_{3}$, and PtOC-as well as $\mathrm{O}$ and $\mathrm{N}$, in charge states $1^{+}$and $2^{+}$, and used the recorded concentrations of carbon in various molecules to distinguish between diamond-dominated and disordered-carbon-dominated regions of acid residue.

We hypothesized that a higher fraction of the disordered carbon would field evaporate as PtOC molecules rather than pure $\mathrm{C}$ molecules, compared to the nanodiamonds because the disordered carbon is more porous than nanodiamond with more exposed surface area where the Pt could bond. Thus, we investigated variations in the concentration of carbon in PtOC versus carbon in $\mathrm{C}_{1}, \mathrm{C}_{2}$, and $\mathrm{C}_{3}$ (using the sum $\mathrm{C}_{1}+2 \times \mathrm{C}_{2}+3 \times \mathrm{C}_{3}$ ) as a potentially useful discriminator between the two carbonaceous fractions. The species $\mathrm{Na}$ and $\mathrm{NaO}$, presumably from the acid dissolution process, are co-located with the carbon clusters, so we included the concentrations of these molecules in our study. We also investigated $\mathrm{O}$ and $\mathrm{N}$ concentrations. Scanning transmission electron microscopy with energy-dispersive $\mathrm{X}$-ray spectrometry (STEM/EDXS) showed that in the acid residue $\mathrm{O}$ is located primarily in the disordered $\mathrm{C}$, and the $\mathrm{N}$ is primarily in the diamond [3]. Trace amounts of $\mathrm{O}$ and $\mathrm{N}$ were detected by APT, although some of this signal is from the Pt matrix in which the acid residue is embedded for APT analysis.

To calculate concentrations, we divided counts of the atoms and molecules of interest by the sum of the counts of all the ions detected in the acid residue regions of interest in the APT reconstructions, including $\mathrm{C}, \mathrm{Na}, \mathrm{Cl}, \mathrm{F}, \mathrm{N}$, and $\mathrm{O}$. We tested the resulting data to ascertain whether the concentrations of these atoms and molecules could be used to distinguish disordered carbon from diamond. Finally, we conducted two analyses to see if there was any correlation between these concentrations and the ${ }^{12} \mathrm{C} /{ }^{13} \mathrm{C}$ ratios of the samples.

\section{Results}

Isolated regions of carbonaceous material a few $\mathrm{nm}$ in size are typically dominated by $\mathrm{C}$, whereas lower density, larger, and less-ordered regions of acid residue material are dominated by PtOC and laboratory contaminants, such as $\mathrm{Na}$ and $\mathrm{NaO}$, along with acid dissolution products $\mathrm{Cl}$ and $\mathrm{F}$. In many cases, high concentrations of $\mathrm{C}$ ions are surrounded by $\mathrm{PtOC}, \mathrm{Na}$, and $\mathrm{NaO}$ (Figure 2). Atom-probe reconstructions of the acid residues with higher $\mathrm{N}$ concentration tend to have higher concentrations of $\mathrm{C}_{1}+\mathrm{C}_{2}+\mathrm{C}_{3}$. However, these are not consistent or strong trends. The highest concentrations of $\mathrm{C}_{1}+2 \times \mathrm{C}_{2}+3 \times \mathrm{C}_{3}$ correspond to the highest N/O ratios (Figure 3). For $\mathrm{C}_{1}+2 \times \mathrm{C}_{2}+3 \times \mathrm{C}_{3}$ concentrations over 0.6 , the minimum N/O ratio increases steadily from $<0.01$ to 0.05 . One out of seven nanotips, or $\sim 14 \%$ of data sets with $\mathrm{C}_{1}+2 \times \mathrm{C}_{2}+3 \times \mathrm{C}_{3}$ concentration $<0.4$, had a N/O ratio $>0.05$, but $\sim 31 \%(9 / 29)$ with $C_{1}+2 \times C_{2}+3 \times C_{3}$ concentration $>0.4$ had a N/O ratio $>0.05$. There was also a loose trend of lower N/O for higher
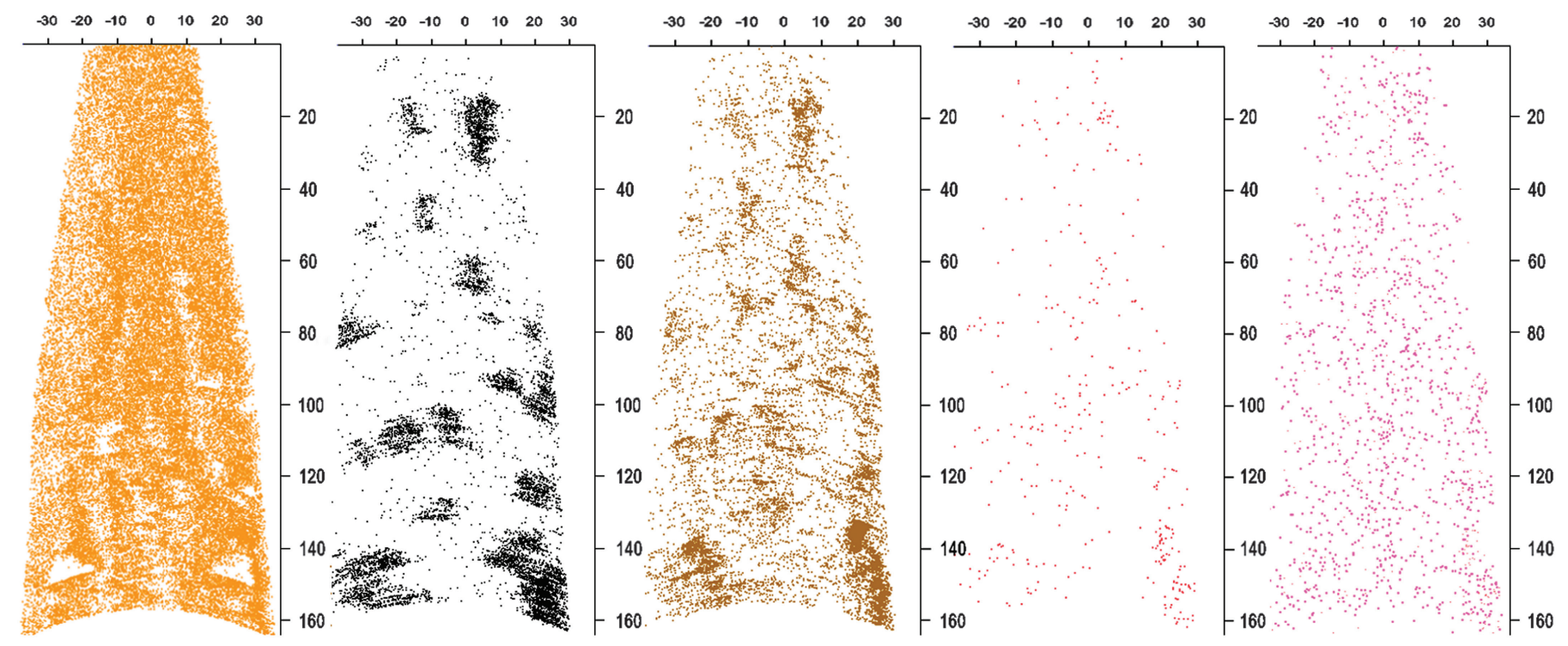

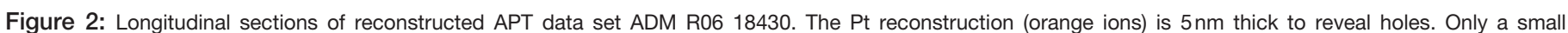

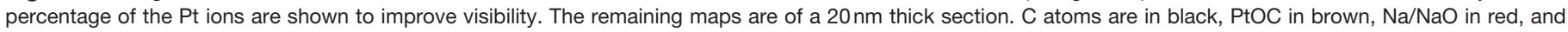

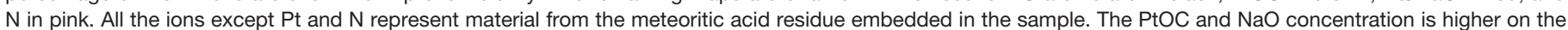

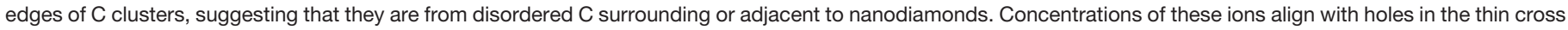
section of $\mathrm{Pt}$. The concentration of $\mathrm{N}$ in the $\mathrm{Pt}$ is too high to distinguish it from $\mathrm{N}$ in the acid residue. Scale bars are in $\mathrm{nm}$. 

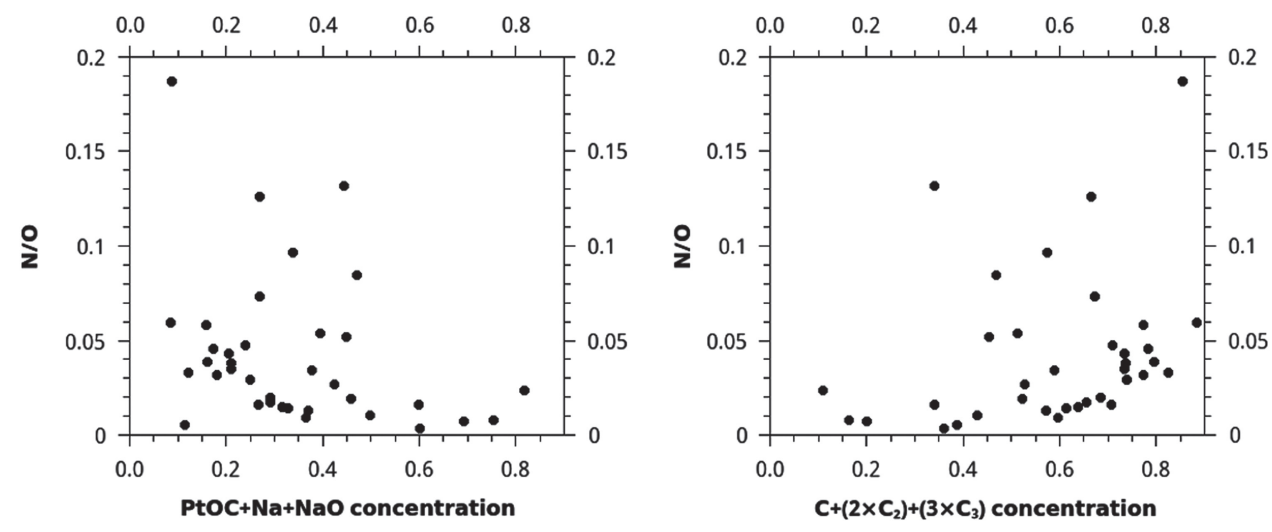

Figure 3: Plots of N/O vs. the concentrations of two different sets of ions in each of 36 APT reconstructed acid residue samples. Concentrations are normalized to the sum of the counts of all ions of interest, including $\mathrm{C}, \mathrm{Na}$, $\mathrm{Cl}, \mathrm{F}, \mathrm{N}$, and $\mathrm{O}$. The very loose trend of higher concentrations of $\mathrm{C}+\left(2 \times \mathrm{C}_{2}\right)+\left(3 \times \mathrm{C}_{3}\right)$ for higher $\mathrm{N} / \mathrm{O}$, and the opposite trend of lower concentrations of $\mathrm{PtOC}+\mathrm{Na}+\mathrm{NaO}$ for higher $\mathrm{N} / \mathrm{O}$, may be explained as a mixture of two phases in the meteoritic material, such as disordered $\mathrm{C}$ and nanodiamonds.
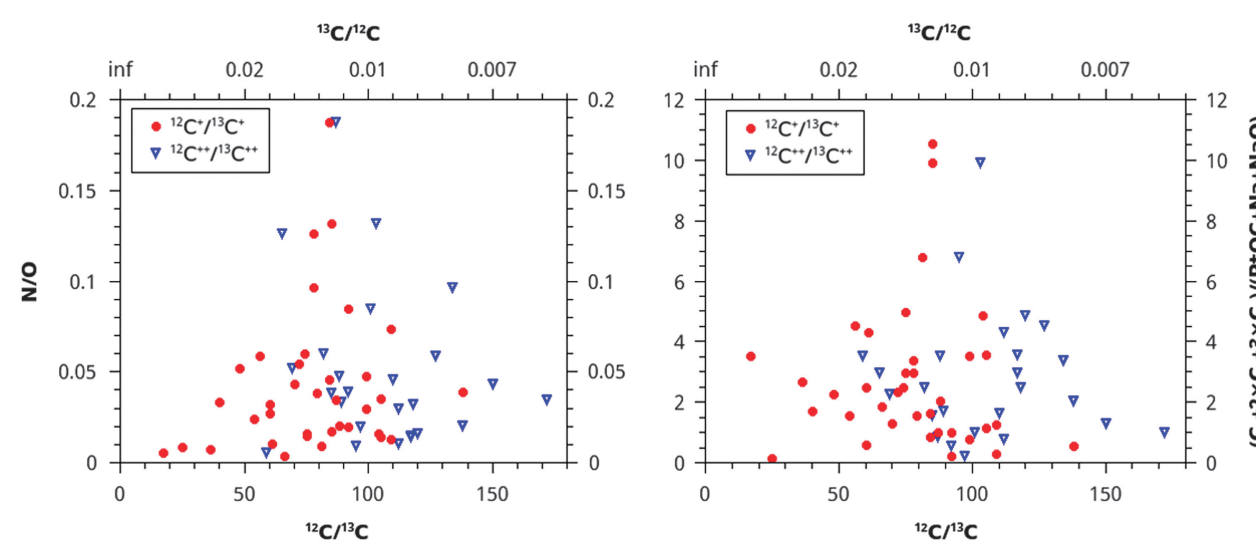

Figure 4: N/O ratio and $\left(\mathrm{C}_{1}+2 \times \mathrm{C}_{2}+3 \times \mathrm{C}_{3}\right) /(\mathrm{PtOC}+\mathrm{Na}+\mathrm{NaO})$ ratios plotted vs. the normalized ${ }^{12} \mathrm{C} /{ }^{13} \mathrm{C}$ isotopic ratios. No trends are present, giving no evidence that more than one isotopic reservoir contributed material to the samples.

PtOC+Na+NaO concentration: $\sim 13 \%(1 / 8)$ of data sets with $\mathrm{PtOC}+\mathrm{Na}+\mathrm{NaO}$ concentration $>0.45$ had $\mathrm{N} / \mathrm{O}>0.05$, whereas $\sim 32 \%(9 / 28)$ with PtOC+Na+NaO concentration $<0.45$ had N/O $>0.05$.

These data suggest that these two ratios record information about a mixture of two phases, such that data sets with extreme values in one of these ratios contain acid residue composed primarily either of disordered $\mathrm{C}$, with $\mathrm{O}$ and $\mathrm{PtOC}+\mathrm{Na}+\mathrm{NaO}$, or of diamond, with $\mathrm{N}$ and $\mathrm{C}_{1}+2 \times \mathrm{C}_{2}+3 \times \mathrm{C}_{3}$. Some of the correlation for $\mathrm{PtOC}+\mathrm{Na}+\mathrm{NaO}$ with $\mathrm{N} / \mathrm{O}$ is due to the fact that $\mathrm{O}$ is present in $\mathrm{PtOC}$ and $\mathrm{NaO}$, both of which are also included in the ion count used to calculate concentration, but, taken along with the lack of $\mathrm{N}$ and higher $\mathrm{O}$ for lower $\mathrm{C}_{1}+2 \times \mathrm{C}_{2}+3 \times \mathrm{C}_{3}$ concentration; these concentrations can be used as a qualitative proxy for the fraction of the acid residue that is nanodiamond versus disordered carbon. This trend is not easily discernable when $\mathrm{N}$ or $\mathrm{O}$ are plotted separately instead of N/O.

Instead of using concentration, we could divide the counts of PtOC by the counts of $\mathrm{C}_{1}+2 \times \mathrm{C}_{2}+3 \times \mathrm{C}_{3}$, and the counts of $\mathrm{N}$ by $\mathrm{O}$. Since deviation from solar ratios of stable isotopes is the best indicator of whether material is presolar, these values were plotted versus the ${ }^{12} \mathrm{C} /{ }^{13} \mathrm{C}$ ratios of the microtips. The results of this analytical method are null; no trends are apparent (Figure 4). disordered $\mathrm{C}$ and that these two phases formed from two different isotopic reservoirs. However, we note that the trends were only apparent for the $1^{+}$ratios. This suggests that rather than inherent isotopic anomalies, these trends are due to an instrumental artifact that affects $1^{+}$ratios more than $2^{+}$, for example, a ${ }^{12} \mathrm{CH}^{+}$hydride interference. This explanation would imply that contamination of the mass spectrum with hydrides, formed from residual gas in the atom-probe analysis vacuum chamber, goes up with increasing fraction of disordered $\mathrm{C}$ (more $\mathrm{O}$ ) and decreasing fraction of diamond (less C). Such contamination would cause lower measured $\mathrm{C}^{+}$ratios due to misindentification of ${ }^{12} \mathrm{CH}^{+}$hydride at $13 \mathrm{amu}$ in the mass spectrum as ${ }^{13} \mathrm{C}^{+}$, but not lower $\mathrm{C}^{2+}$ ratios, since ${ }^{12} \mathrm{CH}^{2+}$ forms much less readily than ${ }^{12} \mathrm{CH}^{+}$.

Future TEM work will be carried out in order to confirm this finding by distinguishing disordered carbon from diamonds in the Pt nanotips, using electron energy loss spectrometry (EELS), prior to APT. The TEM/EELS analysis can also distinguish between different types of bonding in disordered $\mathrm{C}$, allowing us to comment on how specific types of disordered C behave under atom-probe analysis conditions.

\section{Conclusion}

We have identified an approach that allows us for the first time to distinguish qualitatively between APT reconstructions 

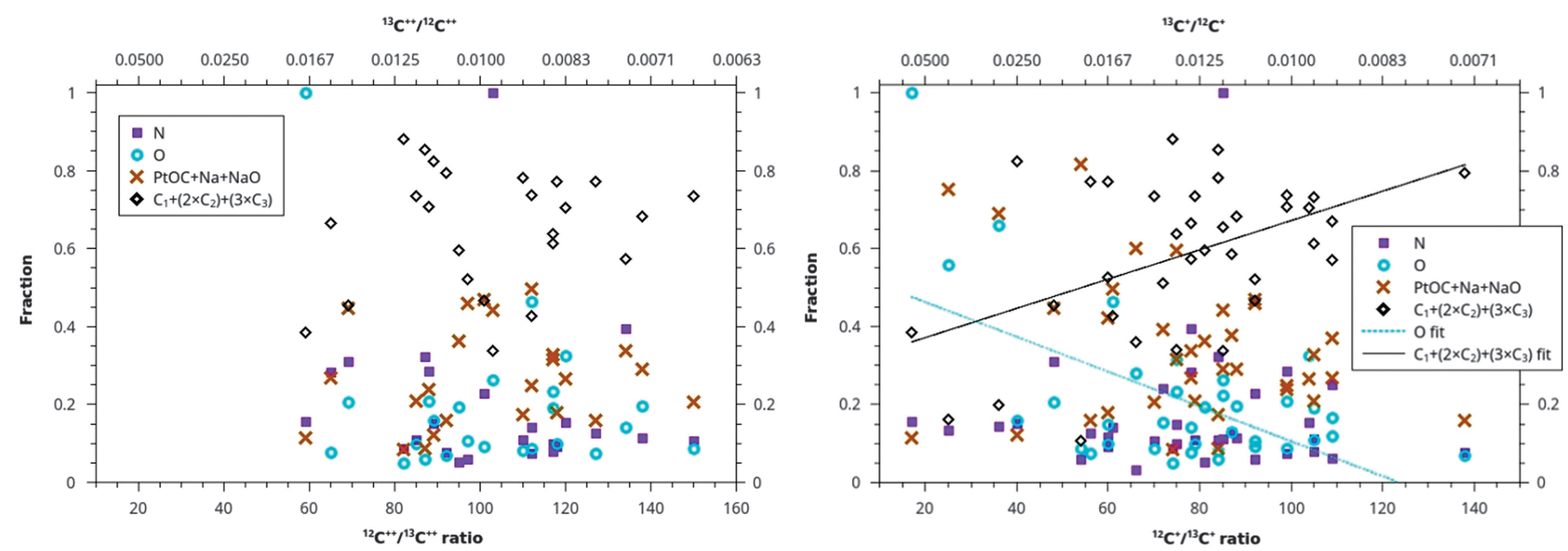

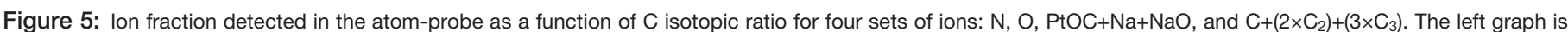

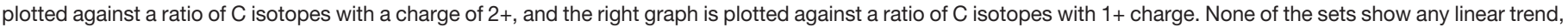

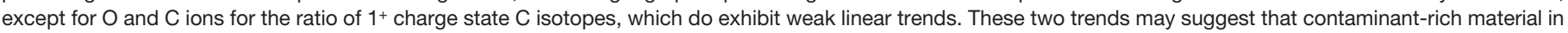

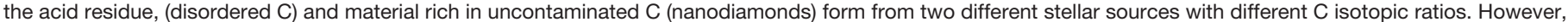
these data may be exhibiting hydride interference (see text).

of acid residue that are dominated either by nanodiamonds or disordered carbon. This analysis is based on previous findings that nitrogen is more likely to form in nanodiamonds.

An initial comparison with the ${ }^{12} \mathrm{C} /{ }^{13} \mathrm{C}$ ratios of the microtips suggests that disordered, probably glassy carbon, has similar ${ }^{12} \mathrm{C} /{ }^{13} \mathrm{C}$ ratios to the nanodiamonds, indicating they may have formed from the same isotopic reservoir. Small differences between the isotopes in the two phases appear to be indicative of hydride formation proceeding more readily on disordered $\mathrm{C}$ than on nanodiamonds.

We expect that complementary or correlated studies by transmission electron microscopy, while potentially more conclusive in its identification of phases, may not always be necessary to distinguish between different material phases in the APT. Contaminant ions, which are more likely to form in each phase, may be used to distinguish phases.

\section{Acknowledgments}

This work is supported by NASA grants NNX14AP15H (J.B.L.) and NNX16AD26G (C.F.). The Cameca LEAP atom probe tomograph at the Northwestern University Center for Atom Probe Tomography (NUCAPT) was acquired and upgraded with NSF DMR-0420532 and ONR-DURIP N00014-0400798, N00014-0610539, N000140910781 equipment grants. NUCAPT received support from the MRSEC program (NSF DMR-1121262) at the Materials Research Center, the SHyNE Resource (NSF NNCI-1542205), and the Initiative for Sustainability and Energy at Northwestern (ISEN)).

\section{References}

[1] RS Lewis et al., Nature 326 (1987) 160-62.

[2] TJ Bernatowicz et al., Astrophys J 359 (1990) 246-55.

[3] RM Stroud et al., Astrophys J Lett 738 (2011) L27-L31.

[4] E Zinner, Presolar Grains. in Treatise on Geochemistry 2nd Ed. (Vol. 1), eds. H Holland and K Turekian, Elsevier, Oxford, UK, 2014.

[5] SS Russell et al., Met and Planet Sci 31 (1996) 343-55.
[6] TL Daulton et al., Geochim et Cosmochim Acta 60 (1996) 4853-72.

[7] PR Heck et al., Met and Planet Sci 49 (2014) 453-67.

[8] D Isheim et al., Microsc Microanal 19 (Suppl 2) (2013) 974-75.

[9] JB Lewis et al., Ultramicroscopy 159 (2015) 248-54.

[10] NR Greiner et al., Nature 333 (1988) 440-42.

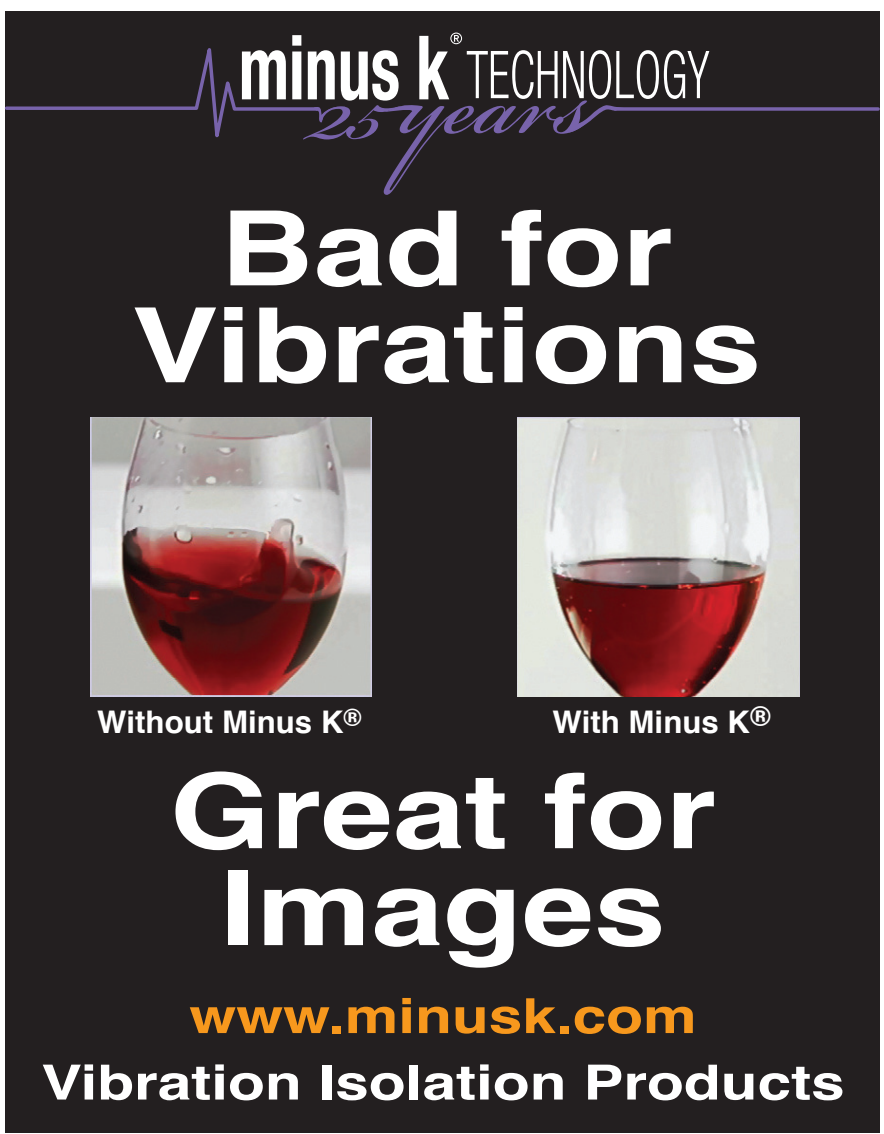




\section{Combining data quality with intuitive operation.}

\section{ZEISS EVO}

\section{// RELIABILITY}

MADE BY ZEISS

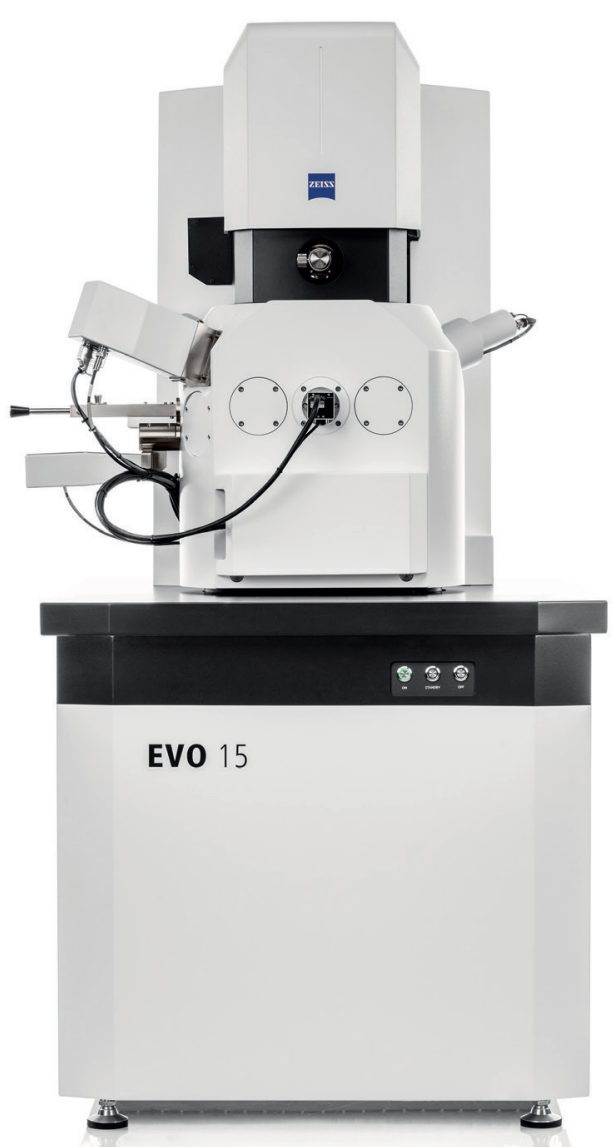

Your modular SEM platform for routine investigations and research applications

The instruments of the EVO family combine high performance scanning electron microscopy with an intuitive, user-friendly experience that appeals to both trained microscopists and new users. With its comprehensive range of available options, EVO can be tailored precisely to your requirements, whether you are in life sciences, material sciences, or routine industrial quality assurance and failure analysis. 